Trauma Berufskrankh 2008 10 [Suppl 1]:120-124

DOI 10.1007/s10039-007-1297-1

Online publiziert: 15. Dezember 2007

(c) Springer Medizin Verlag 2007

\author{
A. Lohsträter ${ }^{1} \cdot$ J. Schmidt ${ }^{2} \cdot$ T. Kohlmann ${ }^{3} \cdot$ A. Ekkernkamp ${ }^{4,5}$ \\ ${ }^{1}$ VBG (Verwaltungs-Berufsgenossenschaft), Erfurt \\ ${ }^{2}$ Klinik für Unfall- Hand- und Wiederherstellungschirurgie, \\ Helios Klinikum Berlin-Buch, Berlin \\ ${ }^{3}$ Institut für Community Medicine, Universitätsklinikum, Greifswald \\ ${ }^{4}$ Unfallkrankenhaus, Berlin \\ ${ }^{5}$ Abteilung für Unfall- und Wiederherstellungschirurgie, \\ Universitätsklinikum, Greifswald
}

\title{
Effizienz der Heilverfahrenssteuerung
}

\section{Hintergrund}

Gerade in der Unfallversicherung ist es das Ziel der Rehabilitation, die Teilhabe der Betroffenen am Arbeitsleben und am Leben in der Gemeinschaft möglichst schnell und dauerhaft wiederherzustellen und zu sichern. Die zu diesem Zweck ergriffenen Maßnahmen zur Wiederherstellung der Gesundheit und Leistungsfähigkeit werden in den letzten Jahren in Deutschland zunehmend unter den Blickwinkeln der Effektivität und der Effizienz betrachtet. Hinzu kommt der mit dem SGB IX an die Rehabilitationsträger gerichtete Auftrag zur Intensivierung der Qualitätssicherung auf diesem Gebiet. Seitens der Unfallversicherungsträger werden Projekte initiiert, um diese Ziele zu erreichen und die Qualität der Verfahren und Ergebnisse zu dokumentieren.

Die Rahmenbedingungen zur Beurteilung von Effektivität und Effizienz werden dabei durch den Gesetzgeber definiert. Die Unfallversicherungsträger haben „mit allen geeigneten Mitteln möglichst frühzeitig... die Gesundheitsschäden zu beseitigen oder zu bessern... und die Folgen zu mildern...“(\$26 Abs. 2 SGB VII)

und dabei die

„Grundsätze der Wirtschaftlichkeit und Sparsamkeit“ ( $\$ 69$ Abs. 2 SGB IV)

zu beachten.

Untersuchungen zur Effizienz in der Heilverfahrenssteuerung können sich beispielhaft orientieren an:
- dem Einsatz von Klassifizierungssystemen,

- dem Einsatz von Kodierungssystemen,

- der Optimierung von Verfahren und Verfahrensschritten,

- der Anwendung von Assessmentinstrumenten oder

- der Bewertung von Kosten (KostenNutzen-Analysen).

Nicht zu vernachlässigen sind bei der Beurteilung der Effizienz in der Heilverfahrenssteuerung nach Ansicht der Autoren die Implementierung von Leitlinien und Therapieempfehlungen inklusive eines integrierten Reha-Managements $[1,3]$.

\section{Material und Methode}

Im Rahmen ihrer seit 2002 durchgeführten Untersuchungen und Studien hat die VBG die nachfolgenden Verfahren überprüft:

- Kosteneffekte des Reha-Managements am Beispiel der Fersenbeinfraktur

- Effizienz und Kosteneffektivität der BGSW (berufsgenossenschaftliche stationäre Weiterbehandlung)

- Notwendigkeit des Einsatzes von Assessmentinstrumenten in der Rehabilitation von Unfallverletzten

- Wertigkeit eines Handreha-Managements
Bei der Untersuchung der isolierten Fersenbeinfrakturen wurden nach dem $\mathrm{Zu}$ fallsprinzip aus dem Datenbestand eine Stichprobe ohne $(n=29)$ mit einer 2. Stichprobe mit Reha-Management $(n=20)$ verglichen [2]. Die Studie zur Untersuchung der Wertigkeit der BGSW wurde als prospektive Studie mit $\mathrm{n}=111$ Patienten durchgeführt. Zu 88 Patienten konnten historische Kontrollen gefunden werden, um die Gesamtfallkosten und die Kostenstruktur zu vergleichen [4]. Im Rahmen ihres Reha-Managements setzt die VBG zur Beurteilung des Rehabilitationserfolges selbstadministrierte Assessmentinstrumente ein [7]. Die Erkenntnis wurde aus Vorstudien gewonnen. Die retrospektiv angelegte Vorstudie zur Beurteilung eines Handreha-Managements zeigte weiteres Untersuchungspotenzial $[5,6]$.

Die randomisiert angelegte Untersuchung des Handreha-Managementverfahrens bildet den Schwerpunkt der vorliegenden Darstellung. Es wurden eine Kontroll- und eine Interventionsgruppe gebildet. In Ersterer wurden die Steuerung und Überwachung des Heilverfahrens sowie das Verordnen der Übungsbehandlung hinsichtlich Art, Intensität und Ausmaß ausschließlich dem behandelnden Arzt überlassen.

In der Interventionsgruppe wurden nach Eingang des Durchgangsarzt- und ggf. des Operationsberichtes die Unterlagen dem beratenden Handchirurgen übermittelt. Dieser gibt innerhalb von $24 \mathrm{~h}$ 
eine dezidierte Behandlungsempfehlung für den weiterbehandelnden Arzt ab. Voraussichtliche Arbeitsunfähigkeitszeiten werden prognostisch angegeben [6]. Die ausführenden Reha-Manager leiten diese Empfehlungen an den Behandler weiter, informieren und beraten die Patienten entsprechend und überwachen die Umsetzung. Legen die behandelnden Ärzte alternative Behandlungsstrategien vor, stimmen die Reha-Manager diese mit dem beratenden Handchirurgen $\mathrm{ab}$ und koordinieren das abschließende Vorgehen.

Die Fallzahlschätzung von 198 Patienten wurde aus der entsprechenden Vorstudie $[5,6]$ gewonnen. Die Frakturklassifikation wurde vom behandelnden Arzt im Erstbericht angegeben oder vom Reha-Manager angefordert. Zur Ergebnissicherung wurden die angegebenen Frakturtypen anhand der vorliegenden Röntgenaufnahmen nachklassifiziert. Diese Ergebnisse werden gesondert publiziert werden. Den Vorgaben der Berichte der gesetzlichen Unfallversicherung folgend, wurde die Klassifikation nach der Arbeitsgemeinschaft für Osteosynthese (Arbeitsgemeinschaft $\mathrm{AO}$ ) genutzt.

Die Signifikanztests wurden als U-Test nach Mann-Whitney (Fallkosten und Arbeitsunfähigkeit) bzw. als $\chi^{2}$-Test (Rente, MdE) mit einem Signifikanzniveau von $5 \%$ durchgeführt.

\section{Ergebnisse}

\section{Studiengröße}

Im Zeitraum von Oktober 2002 bis einschließlich Januar 2006 konnten 380 Patienten randomisiert werden. Aufgrund unterschiedlicher Drop-outs bildeten 198 Patienten, aufgeteilt in beide Gruppen, die empirische Basis der Studie.

\section{Kostenverteilung}

Die niedrigsten Gesamtfallkosten betrugen 271,13 EUR bei einer AO-23B1-Fraktur. Die höchsten Gesamtfallkosten für eine distale Radiusfraktur in der Studie betrugen rund 137.00o EUR ( $\mathrm{AO}_{23} \mathrm{C}_{2}$ ). Die auf die Studienarme bezogene Verteilung der Gesamtfallkosten ist $\bullet$ Abb. 1 zu entnehmen. Diese ließen sich aufteilen in:

Trauma Berufskrankh 2008 • 10[Suppl 1]:120-124 DOI 10.1007/s10039-007-1297-1

(C) Springer Medizin Verlag 2007

\section{A. Lohsträter · J. Schmidt · T. Kohlmann · A. Ekkernkamp Effizienz der Heilverfahrenssteuerung}

\section{Zusammenfassung}

Die vorliegende Arbeit führt am Beispiel der gesetzlichen Unfallversicherung VBG unterschiedliche Untersuchungen zur systematischen Steuerung von Behandlungsabläufen an und zeigt auszugsweise erste Ergebnisse zur Wirksamkeit von Managementverfahren bei der distalen Radiusfraktur. In allen Untersuchungen wurden Patienten der VBG, nach für die jeweilige Studie festgelegten Parametern, eingeschlossen und analysiert. Teilweise kamen Assessmentinstrumente zum Einsatz. Mit adäquaten Managementverfahren konnte, auch unter Betrachtung von Outcomeparametern, die Effektivität des berufsgenossenschaftlichen Heilverfahrens im Sinne der Effizienz gesteigert werden. In der Studie zur Beurteilung des Handreha-Managements konnten in der Interventionsgruppe

\section{Efficiency of treatment controlling}

\section{Abstract}

This study presents various investigations into systematic control of treatment development and initial results of a study on the effectiveness of management methods in the treatment of distal radius fractures. VBG patients were included and evaluated in all studies. Assessment instruments were used in some investigations. The effectiveness of BG treatment procedures in the sense of efficiency could be improved with adequate management procedures, even when outcome parameters were considered. Average costs and duration of unfitness for work following accident were reduced in the intervention group in the study on the evaluation of the management of rehabilitation following procedures on the hand. Higher ther- die durchschnittlichen Kosten, die Dauer der unfallbedingten Arbeitsunfähigkeit sowie die Anzahl der Fälle mit einer Minderung der Erwerbsfähigkeit reduziert werden. Höhere Therapiekosten waren in der Interventionsgruppe nicht zu beobachten. Aus der Kostenanalyse kann geschlossen werden, dass das Handreha-Management zu optimierten ökonomischen Ergebnissen bei der Heilverfahrenssteuerung der Unfallversicherungsträger beiträgt. In dieser Hinsicht war die Behandlungssteuerung im Kontext des HandrehaManagements effektiv und effizient.

\section{Schlüsselwörter}

Qualitätssicherung · Effizienz · Strukturierte Nachbehandlung · Managementverfahren . Handreha-Management apy costs were not observed in the intervention group. Consideration of the costs allows the conclusion that hand rehabilitation management contributes to optimized economic results in the course of controlling of treatments by the underwriters of accident insurance companies. In this sense, treatment controlling in the context of hand rehab management was found to be effective and efficient.

\section{Keywords}

Quality assurance $\cdot$ Efficiency $\cdot$ Structured aftertreatment - Management procedures Management of rehabilitation following procedures on the hand 

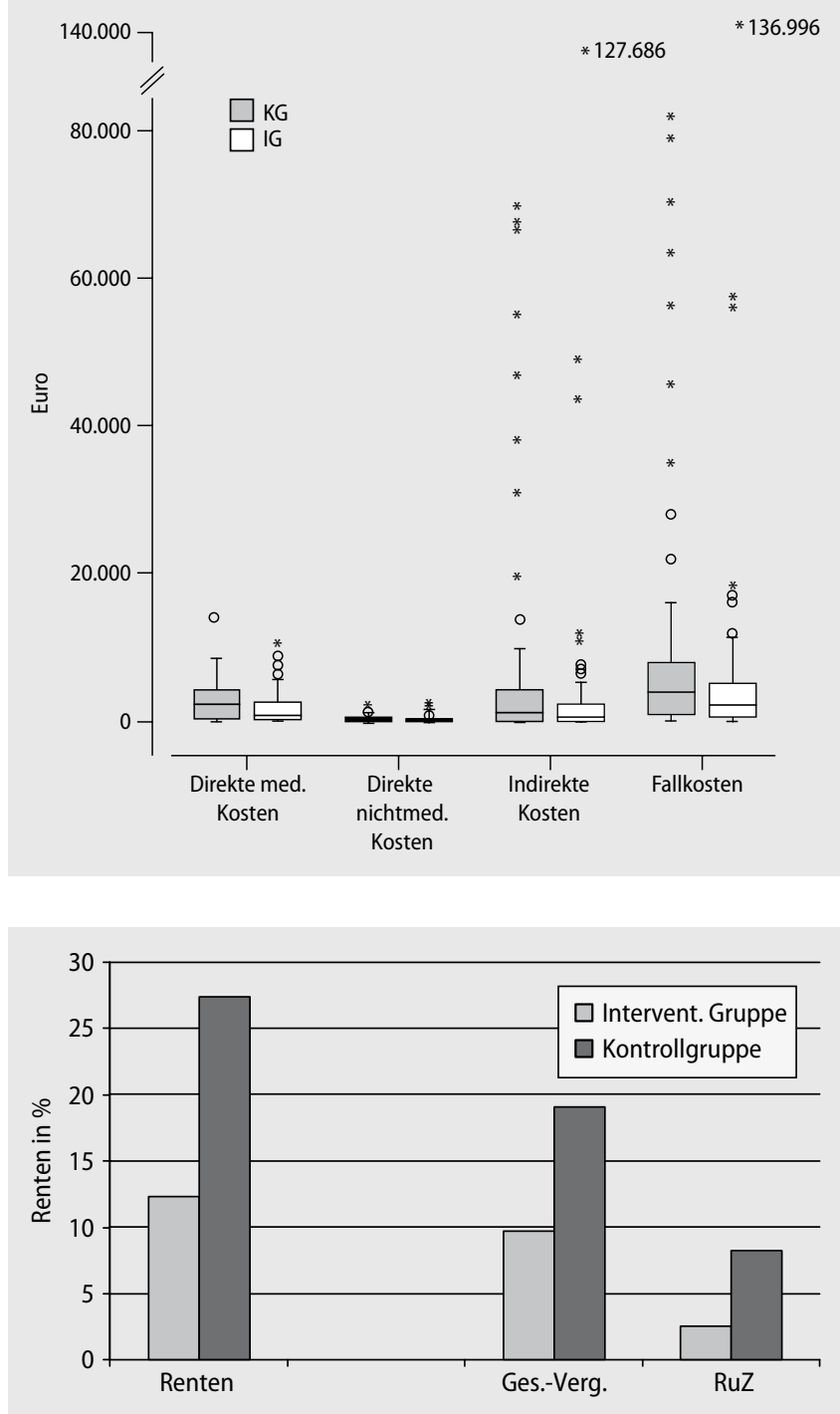

Abb. $1 \varangle$ Kostenverteilung

Abb. $2 \varangle$ Renten gesamt, Ges.-Verg. Gesamtvergütung, RuZ Rente auf unbestimmte Zeit

- direkte medizinische Kosten,

- direkte nichtmedizinische Kosten und - indirekte Kosten.

Die direkten medizinischen Kosten waren die Kosten für die stationäre Behandlung (soweit durchgeführt), Übungsbehandlung, Hilfsmittel und Kosten der ärztlichen Behandlung. Sie betrugen in der Interventionsgruppe 1793,47 EUR und in der Kontrollgruppe 2755,79 EUR.

Direkte nichtmedizinische Kosten waren Fahrtkosten und Berichtsgebühren. Durch die Steuerung im Verfahren ergaben sich keine höheren direkten nichtmedizinischen Kosten in der Interventionsgruppe.

Indirekte Kosten waren als Verletztengelder und Renten definiert. Hier waren erwartungsgemäß die größten Unterschiede zwischen den Gruppen zu ver- zeichnen. Die Mittelwerte betrugen in der Interventionsgruppe 3346,37 EUR; in der Kontrollgruppe 6513,34 EUR.

Die Verteilung der Kosten in $\bullet$ Abb. 1 zeigt für die Interventionsgruppe durchgängig niedrigere Werte in allen Kostengruppen. Ebenso ist eine homogenere Verteilung mit weniger Ausreißern festzustellen. Bereits bei dieser Betrachtung sind erste Hinweise auf die Wirksamkeit des Handreha-Managements festzuhalten.

- Tab. 1 bildet die Verteilung der Gesamtfallkosten bezogen auf die jeweiligen Frakturtypen im Gruppenvergleich ab. Der Signifikanztest wurde als nichtparametrischer Test durchgeführt (U-Test nach Mann-Whitney) und zeigte aufgrund der hohen Standardabweichung ausschließlich bei den C-Frakturen das gewählte Signifikanzniveau. Betrachtet man die Kostenvorteile in der Interven- tionsgruppe bei allen Frakturtypen, die mehrere tausend Euro je Fall ausmachen, dürfte der Wert der signifikanten Unterschiede zwischen den Gruppen allerdings in den Hintergrund treten.

Publizierte Daten aus randomisierten Studien zur distalen Radiusfraktur mit vergleichbarer Kostenstruktur liegen nicht vor. Ein Literaturvergleich war daher nicht möglich.

\section{Zeiten der Arbeitsunfähigkeit}

Die durchschnittliche Dauer der Arbeitsunfähigkeit betrug in der Interventionsgruppe 60,2 Tage. In der Kontrollgruppe trat die Arbeitsfähigkeit der Patienten durchschnittlich nach 92,6 Tagen und damit deutlich später als in der Interventionsgruppe ein $(p=0,007)$. Einzelheiten zur Dauer der Arbeitsunfähigkeit, auch bezogen auf die jeweilige Frakturklassifikation, sind $\bullet$ Tab. 2 zu entnehmen.

\section{Minderung der Erwerbsfähigkeit und Renten}

Im Outcome, gemessen in der Minderung der Erwerbsfähigkeit (MdE), zeigte sich, trotz kürzerer Arbeitsunfähigkeitszeit, in der Interventionsgruppe nur in 12,3\% der Fälle eine rentenberechtigende MdE. In der Kontrollgruppe wurde, trotz scheinbar längerer Nachbehandlungsphase, in $27,4 \%$ der Fälle eine MdE festgestellt

\section{(• Abb. 2).}

Der Anteil an Gesamtvergütungen betrug in der Interventionsgruppe 9,7\% und in der Kontrollgruppe 19,1\%. Renten auf unbestimmte Zeit waren in der Interventionsgruppe in 2,6\% der Fälle vorhanden, in der Kontrollgruppe in $8,3 \%$ der Fälle ( $\mathrm{p}=0,022 \mathrm{im} \chi^{2}$-Test). In der Interventionsgruppe kamen 9 von den 11 zu gewährenden Gesamtvergütungen bei CFrakturen vor und jeweils eine bei einer A- bzw. einer B-Fraktur. In der Kontrollgruppe wurden Gesamtvergütungen bei 2 A-, 5 B- und 9 C-Frakturen beobachtet.

Laufenden Renten waren in der Interventionsgruppe $(n=3)$ ausschließlich bei C-Frakturen auszuweisen. In der Kontrollgruppe waren sie bei 2 A-, 2 B- und 3 C-Frakturen festzustellen. 
Das Outcome in der Interventionsgruppe ist als besser, d. h. mit prozentual weniger Rentenfällen, zu klassifizieren.

\section{Diskussion}

Nach der Entwicklung und Einführung des Handreha-Managementverfahrens [6] sowie der Publikation erster Teilergebnisse [5] bedurfte es einer randomisierten Studie [9], um die Resultate zu untermauern. Studiengröße und -durchführung sind belastbar.

Bei Vergleichbarkeit der Schweregrade der Verletzungen in beiden Gruppen waren durchgängig Vorteile zugunsten der Interventionsgruppe festzustellen.

Die Hypothese einer kürzeren Arbeitsunfähigkeitsdauer zugunsten der Interventionsgruppe konnte, statistisch signifikant, bestätigt werden, ebenso die Hypothese zur Häufigkeit der Minderung der Erwerbsfähigkeit (Outcome). Auch hier konnte nachgewiesen werden, dass das Outcome in der Interventionsgruppe signifikant besser ist als in der Kontrollgruppe. Das Outcome in der Kontrollgruppe entsprach weitestgehend den publizierten Feststellungen anderer Kostenträger [8].

Die hypothetisch angenommenen niedrigeren Gesamtfallkosten in der Interventionsgruppe ließen sich bereits durch die kürzeren Arbeitsunfähigkeitszeiten und niedrigen Rentenquoten verifizieren.

Die spezifische Betrachtung der ökonomischen Größen bestätigte die aufgestellte Hypothese eines effizienten Managementverfahrens. Die indirekten Kosten (Verletztengeld und Unfallrenten) machten in beiden Gruppen etwa 2/3 der Gesamtkosten aus. Die Ausgaben für Verletztengeld waren bei allen Frakturtypen in der Interventionsgruppe aufgrund der kürzeren Dauer der Arbeitsunfähigkeit geringer. Den größten Kostenanteil hatten die zu gewährenden Renten. In der Interventionsgruppe waren deutlich weniger Renten (Interventionsgruppe: 12,3\%, Kontrollgruppe: $\mathbf{2 7 , 4 \% )}$ festzustellen. Laufende Renten kamen in der Interventionsgruppe ausschließlich bei den vollständig artikulären Frakturen vor. In der Kontrollgruppe waren laufende Renten über alle Frakturtypen verteilt zu finden.

\begin{tabular}{|c|c|c|c|}
\hline Frakturtyp & Mittelwert [EUR] & Standardabweichung [EUR] & p-Wert ${ }^{a}$ \\
\hline \multicolumn{4}{|l|}{ A-Frakturen } \\
\hline Kontrollgruppe & 6121,76 & $15.155,92$ & \multirow[t]{2}{*}{0,994} \\
\hline Interventionsgruppe & 2799,97 & 3138,30 & \\
\hline \multicolumn{4}{|l|}{ B-Frakturen } \\
\hline Kontrollgruppe & 6977,09 & $13.908,72$ & \multirow[t]{2}{*}{0,136} \\
\hline Interventionsgruppe & 2074,01 & 2312,02 & \\
\hline \multicolumn{4}{|l|}{ C-Frakturen } \\
\hline Kontrollgruppe & $11.435,01$ & $16.394,95$ & \multirow[t]{2}{*}{0,045} \\
\hline Interventionsgruppe & 7775,45 & $12.509,83$ & \\
\hline
\end{tabular}

Tab. 2 Dauer der Arbeitsunfähigkeit bezogen auf die Frakturklassifikation

\begin{tabular}{|lll}
\hline $\begin{array}{l}\text { Frakturtyp } \\
\text { Gesamt }\end{array}$ & Mittelwert [Tage] & Standardabweichung [Tage] \\
\hline Kontrollgruppe & 92,6 & 105,8 \\
\hline Interventionsgruppe & 60,2 & 38,8 \\
\hline A-Frakturen & & \\
\hline Kontrollgruppe & 91,2 & 147,3 \\
\hline Interventionsgruppe & 49,5 & 36,3 \\
\hline B-Frakturen & & \\
\hline Kontrollgruppe & 64,9 & 39,8 \\
\hline Interventionsgruppe & 48,3 & 19,2 \\
\hline C-Frakturen & & \\
\hline Kontrollgruppe & 113,5 & 106,6 \\
\hline Interventionsgruppe & 81,6 & 45,2 \\
\hline
\end{tabular}

Die Ergebnisse zeigen einen hohen Wirkungsgrad des Handreha-Managementverfahrens der VBG, dessen Bestandteil auch eine Empfehlung zum kombinierten Einsatz von Physio- und Ergotherapie ist. Managementverfahren dieser Art werden hinsichtlich ihrer Auswirkungen und ihrer Bedeutung noch unterschätzt.

\section{Schlussfolgerung}

Ziel dieser Untersuchung war, die Effektivität und Effizienz des Handreha-Managements der VBG zu überprüfen. Festzuhalten bleibt, dass auf die im Rahmen des berufsgenossenschaftlichen Heilverfahrens bestehenden Regelungen als Grundvoraussetzungen nicht verzichtet werden kann. Sie erreichen allerdings einen noch höheren Wirkungsgrad, wenn der das Heilverfahren führende UV-Träger Managementverfahren weiterentwickelt und mit seinen entsprechend qualifizierten Reha-Managern aktiv zielgerichtete Handlungsbeiträge erbringt. Eine unterstützende Steuerung scheint daher ge- boten. Die interdisziplinäre Zusammenarbeit zwischen UV-Trägern, Ärzten und Therapeuten ist dabei in einem höheren Maß gefordert als bisher.

Die Zeiten der unfallbedingten Arbeitsunfähigkeiten und das Outcome (MdE) waren in der Interventionsgruppe signifikant kürzer bzw. besser. Höhere Therapiekosten waren, auch ohne Budgetschranken, in der Interventionsgruppe nicht zu beobachten.

Aus der Betrachtung der Kosten kann geschlossen werden, dass das HandrehaManagement $\mathrm{zu}$ optimierten ökonomischen Ergebnissen bei der Heilverfahrenssteuerung der Unfallversicherungsträger beiträgt. In diesem Sinne war die Behandlungssteuerung im Kontext des Handreha-Management effektiv und effizient. Bei deutlich geringeren Kosten konnte ein besseres Outcome erreicht werden. Das Erreichen dieser Ziele war ein Zweck des dargestellten HandrehaManagements der VBG.

Weiterführende Studien erscheinen zur Sicherung und zum Ausbau der Ergebnisse sinnvoll und notwendig. Mit de- 
finierten Studien, auch zu weiteren Diagnosen, wird es weitere Belege für das hohe Niveau der Behandlung und der qualitätsgesicherten Verfahren der gesetzlichen Unfallversicherung geben.

\section{Korrespondenzadresse}

\section{Dr. A. Lohsträter}

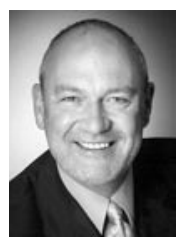

VBG (Verwaltungs-

Berufsgenossenschaft) Erfurt

Koenbergkstraße 1,

99084 Erfurt

Axel.Lohstraeter@vbg.de

Interessenkonflikt. Der korrespondierende Autor gibt an, dass kein Interessenkonflikt besteht.

\section{Literatur}

1. Boldt C, Grill E, Winter S et al. (2004) Einsatz standardisierter Erhebungsinstrumente in der Frührehabilitation. Phys Rehab Med Kuror 14: 18-25

2. Froese E, Wenzel G (2003) Kosten und Qualitätseffekte des dialogischen Reha-Managements. Die BG 5: 203-207

3. Grill E, Harder M, Fischbacher L et al. (2005) Identification of relevant ICF categories by patients in early post-acute rehabilitation facilities. Phys Med Rehab Kuror 15: 168-173

4. Kropf U, Müller WD, Lohsträter A et al. (2006) Effizienz und Kosteneffektivität der Berufsgenossenschaftlichen Stationären Weiterbehandlung (BGSW) nach Verletzungen der Wirbelsäule und der unteren Extremität. Phys Med Rehab Kuror 16: 160-165

5. Lohsträter A, Froese E (2005) Ergebnisse des RehaManagements der Verwaltungs-Berufsgenossenschaft. Phys Med Rehab Kuror 15: 119-123

6. Lohsträter A, Schmidt J (2002) Rehabilitationsmanagement bei Hand- und Handgelenksverletzungen aus Sicht des UV-Trägers. Trauma Berufskrankh 4: 163-167

7. Lohsträter A, Froese E, Haider E et al. (2007) Der Einsatz von Assessmentinstrumenten in der Rehabilitation von Unfallverletzten. Gesundheitswesen 69: 45-49

8. Radek E, Köhler T, Münch K et al. (2003) Verletztenrenten auf unbestimmte Zeit nach distalen Radiusfrakturen. Trauma Berufskrankh 5: 253-259

9. Stengel D, Bauwens K, Ekkernkamp A (2003) Unfallchirurgische Interventionsstudien: randomisiert oder nicht-randomisiert? Unfallchirurg 106: 294299 